\title{
Very Degenerate Higgsino Dark Matter
}

\author{
Eung Jin Chun, ${ }^{a}$ Sunghoon Jung ${ }^{b, c}$ and Jong-Chul Park ${ }^{d}$ \\ ${ }^{a}$ Korea Institute for Advanced Study, \\ Seoul 130-722, Korea \\ ${ }^{b} S L A C$ National Accelerator Laboratory, \\ Menlo Park, CA 94025, U.S.A. \\ ${ }^{c}$ Kavli Institute for Theoretical Physics, \\ Santa Barbara, CA 93106, U.S.A. \\ ${ }^{d}$ Department of Physics, Chungnam National University, \\ Daejeon 34134, Korea \\ E-mail: ejchun@kias.re.kr, shjung@slac.stanford.edu, jcpark@cnu.ac.kr
}

AbStract: We present a study of the Very Degenerate Higgsino Dark Matter (DM), whose mass splitting between the lightest neutral and charged components is $\mathcal{O}(1) \mathrm{MeV}$, much smaller than radiative splitting of $355 \mathrm{MeV}$. The scenario is realized in the minimal supersymmetric standard model by small gaugino mixings. In contrast to the pure Higgsino DM with the radiative splitting only, various observable signatures with distinct features are induced. First of all, the very small mass splitting makes (a) sizable Sommerfeld enhancement and Ramsauer-Townsend (RT) suppression relevant to $\sim 1 \mathrm{TeV}$ Higgsino DM, and (b) Sommerfeld-Ramsauer-Townsend effect saturate at lower velocities $v / c \lesssim 10^{-3}$. As a result, annihilation signals can be large enough to be observed from the galactic center and/or dwarf galaxies, while the relative signal sizes can vary depending on the locations of Sommerfeld peaks and RT dips. In addition, at collider experiments, stable chargino signatures can be searched for to probe the model in the future. DM direct detection signals, however, depend on the Wino mass; even no detectable signals can be induced if the Wino is heavier than about $10 \mathrm{TeV}$.

Keywords: Supersymmetry Phenomenology

ArXiv EPRINT: 1607.04288 


\section{Contents}

1 Introduction 1

2 Very Degenerate Higgsino DM 2

3 Indirect detection of annihilation signals 4

3.1 SRT effects with very small mass splitting 4

3.2 Annihilations at GC and DG 7

4 Other constraints $\quad 10$

$\begin{array}{lll}4.1 \text { Direct detection } & 10\end{array}$

$\begin{array}{lll}4.2 & \text { Collider searches } & 11\end{array}$

$\begin{array}{lll}4.3 \text { Cosmological constraints } & 11\end{array}$

5 Summary and discussions $\quad 12$

\section{Introduction}

The pure Higgsino (with the electroweak-radiative mass splitting $\Delta m=355 \mathrm{MeV}$ between its lightest neutral and charged components) is an attractive candidate of thermal dark matter (DM) for its mass around $1 \mathrm{TeV}$ [1]. As null results at Large Hadron Collider (LHC) experiments push supersymmetry (SUSY) to TeV scale, such Higgsino as the lightest supersymmetric particle (LSP) has recently become an important target for future collider [2-7] and DM search experiments [5-11]. A priori, the Higgsino mass $\mu$ and gaugino masses $M_{1}, M_{2}$ for the Bino and Wino are not related; thus, the pure Higgsino scenario with much heavier gauginos is possible and natural by considering two distinct Peccei-Quinn and $\mathrm{R}$ symmetric limits.

It is, however, difficult to test the pure Higgsino LSP up to $1-2 \mathrm{TeV}$ at collider experiments (including future $100 \mathrm{TeV}$ options) and dark matter detections. Standard collider searches of the pure Higgsino LSP based on jet plus missing energy become hard as final state visible particles become too soft to be well observed due to the small mass splitting [2-4]; but the splitting is still large enough for charged Higgino components to decay promptly at colliders so that disappearing track and stable chargino searches are not able to probe $[2,12]$. Furthermore, the purity of the Higgsino states suppresses DM direct detection signals. DM indirect detection signals are also not large enough because of relatively weak interactions and negligible Sommerfeld enhancements [8-11, 13, 14]. In contrast, the pure Wino DM with the radiative mass splitting of $164 \mathrm{MeV}$, another thermal DM candidate for its mass $\sim 3 \mathrm{TeV}$, provides several ways to test: monojet plus missing energy due to more efficient recoil and larger cross-section $[2,4,15,16]$, disappearing track due to 
the longer-lived charged Wino $[2,15,16]$, and indirect detection due to somewhat stronger interaction and larger enhancement $[8-11,14,17]$. One of the key features of the Wino DM affecting all of these signals is the smaller mass splitting.

It has been noticed that non-perturbative effects can be sizable for the heavy electroweak dark matter annihilation, leading to not only the Sommerfeld enhancement $[13,14]$ but also the Ramsauer-Townsend (RT) suppression [9, 11, 18, 19] that become more evident for smaller mass splitting (or equivalently heavier DM) and higher multiplets (or stronger electroweak interactions) $[9,11]$. The Higgsino-gaugino system, consisting of the weak singlet, doublet and triplet, with variable mass splitting provides a natural framework realizing drastic Sommerfeld-Ramsauer-Townsend (SRT) effects in dark matter annihilations.

This motivates us to investigate a possibility of a very degenerate Higgsino DM whose mass splitting is much smaller than the electroweak-induced $355 \mathrm{MeV}$, realized in the limit of $\mu \ll M_{1,2}$ admitting slight gaugino mixtures. The Higgsino is more susceptible to nearby gauginos than the gaugino is to others as heavier gaugino effects on the Higgsino decouple less quickly: their effects are captured by dimension- 5 operators, while effects on the gaugino DM is captured by dimension-7 operators [10]. Thus, it leads to a plausible situation that heavier gauginos are almost decoupled leaving some traces only in the Higgsino DM sector in spite of a large hierarchy between them. The Very Degenerate Higgsino DM turns out to produce distinct features in indirect detection signals from the galactic center (GC) and dwarf spheroidal satellite galaxies (DG), which can be observed in the near future.

This paper is organized as follows. In section 2, we look for the Higgsino-gaugino parameter space realizing the Very Degenerate Higgsino LSP. In section 3, indirect signals of DM annihilation are studied to feature the SRT effect, which leads to distinct predictions for GC and DG. In section 4, we consider other constraints from direct detection, collider searches, and cosmology. We finally conclude in section 5 .

\section{Very Degenerate Higgsino DM}

We discuss the SUSY parameter space of the Very Degenerate Higgsino DM, which involves the Higgsino mass parameter $\mu$, the Bino and Wino masses $M_{1,2}$, the ratio of the Higgs vacuum expectation values $t_{\beta} \equiv \tan \beta=v_{u} / v_{d}$, the weak mixing angle given by $s_{W} \equiv \sin \theta_{W} \approx$ 0.23 , and the $W$ gauge boson mass $m_{W}$. We assume the limit $\left|M_{1} \pm M_{2}\right|,\left|M_{2} \pm \mu\right|,\left|\mu \pm M_{1}\right| \gg$ $m_{W}$ and $\left|M_{1}\right|,\left|M_{2}\right| \gg|\mu|$. We keep the signs of mass eigenvalues and make eigenvectors real. Later on, we will assume $M_{2}, \mu>0$ and $M_{1}<0$ for the Very Degenerate Higgsino DM, but we will be agnostic about how such signs can be obtained. Meanwhile, all sfermions and heavy Higgs bosons are assumed to be very heavy and not relevant to our study.

Higgsino mass eigenvalues at tree-level are [20, 21],

$$
\begin{aligned}
m_{\chi^{+}} & \simeq|\mu|-\operatorname{sgn}\left(\mu M_{2}\right) \frac{m_{W}^{2}}{\left|M_{2}\right|} s_{2 \beta}>0, \\
m_{\chi_{S, A}^{0}} & \simeq \mp \mu-\frac{m_{W}^{2}}{2 M_{2}}\left(1 \mp s_{2 \beta}\right) \epsilon_{K}, \quad \epsilon_{K} \equiv\left(1+\frac{M_{2}}{M_{1}} t_{W}^{2}\right),
\end{aligned}
$$

where $s_{2 \beta}=\sin 2 \beta$ and so on. The subscripts $S, A$ imply that the mass eigenstates are $\chi_{S, A}^{0} \sim\left(\widetilde{H}_{d}^{0} \pm \widetilde{H}_{u}^{0}\right) / \sqrt{2}$. Which of $\chi_{S}^{0}$ or $\chi_{A}^{0}$ is the LSP depends on the relative sign of $\mu$ 
and $\epsilon_{K} M_{2}$ : the $\chi_{A}^{0}$ is the LSP if the relative sign is positive, and vice versa. Expressing both possibilities, we write the LSP mass as

$$
m_{\chi_{1}^{0}} \simeq \operatorname{sgn}\left(\epsilon_{K} M_{2}\right)\left(|\mu|-\frac{m_{W}^{2}}{2\left|M_{2}\right|}\left(1+\operatorname{sgn}\left(\mu \epsilon_{K} M_{2}\right) \cdot s_{2 \beta}\right)\left|\epsilon_{K}\right|\right) .
$$

Higgsino mass splitting at tree-level is then

$$
\begin{aligned}
\Delta m_{\text {tree }} & \equiv m_{\chi^{+}}-\left|m_{\chi_{1}^{0}}\right| \\
& \simeq-\operatorname{sgn}\left(\mu M_{2}\right) \frac{m_{W}^{2}}{\left|M_{2}\right|} s_{2 \beta}+\frac{m_{W}^{2}}{2\left|M_{2}\right|}\left(1+\operatorname{sgn}\left(\mu \epsilon_{K} M_{2}\right) \cdot s_{2 \beta}\right)\left|\epsilon_{K}\right| .
\end{aligned}
$$

The physical mass splitting is $\Delta m=\Delta m_{\text {tree }}+\Delta m_{\text {loop }}$, where the model-independent electroweak loop corrections give $\Delta m_{\text {loop }} \approx 355 \mathrm{MeV}$ for the Higgsino [12].

Notably, the $\Delta m_{\text {tree }}$ can be negative, so that the resulting physical mass splitting $\Delta m$ can be smaller than the $\Delta m_{\text {loop }}{ }^{1}$ From the above approximations, we find that one way to obtain negative $\Delta m_{\text {tree }}$ is to satisfy the following conditions:

- $\operatorname{sign}\left(\mu M_{2}\right)>0$ is required because only the first term in eq. (2.4) can be negative. Assuming $\mu, M_{2}>0$ from now on, we rewrite

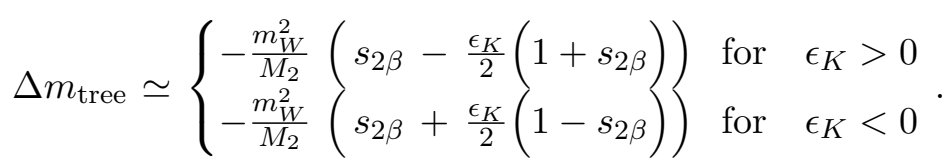

Thus, $\Delta m_{\text {tree }}<0$ if the mass parameters satisfy

$$
-\frac{2 s_{2 \beta}}{1-s_{2 \beta}} \lesssim \epsilon_{K} \lesssim \frac{2 s_{2 \beta}}{1+s_{2 \beta}} \leq 1
$$

- $M_{1}<0$ is preferred so that $\epsilon_{K}<1$. We assume $M_{1}<0$.

- Small $t_{\beta}$ is preferred; $t_{\beta} \gtrsim 2$ does not allow solutions for $\Delta m_{\text {tree }}<0$ for the range of mass parameters considered.

- With the above conditions, no solutions for $\Delta m_{\text {tree }}<0$ exist in the limit of $\left|M_{1}\right| \rightarrow \infty$ or $\left|M_{2}\right| \rightarrow \infty$. We consider $\left|M_{1}\right|,\left|M_{2}\right| \leq 15 \mathrm{TeV}$.

We apply this set of approximate conditions to our full numerical calculation to narrow down the solution finding procedure.

In figure 1, we show one set of numerical solutions for $\Delta m_{\text {tree }}<0$ for the range of $\mu \leq 2 \mathrm{TeV}$ and $-2.5 \geq M_{1} \geq-5 \mathrm{TeV}$ with fixed benchmark parameters $M_{2}=10 \mathrm{TeV}$ and $t_{\beta}=1.8$. In most of the parameter space shown, $\Delta m$ is smaller than the radiative mass splitting of $355 \mathrm{MeV}$. Although the approximate equations above do not depend on $\mu$, the full numerical solution does a bit. We will consider two benchmark cases of $\Delta m=2$, $10 \mathrm{MeV}$ in this parameter space throughout. Later, we will also comment on the case with smaller $M_{2}=5 \mathrm{TeV}$. The solutions for $\Delta m=2,10 \mathrm{MeV}$ and our most discussions do not strongly depend on the value of $M_{2}$, but direct detection signals do as will be discussed. The neutralino mass splitting, $\delta m^{0} \equiv\left|m_{\chi_{2}}^{0}\right|-\left|m_{\chi_{1}}^{0}\right|$, is somewhat larger $\sim \mathcal{O}(100) \mathrm{MeV}$, and it also does not strongly affect our discussion.

\footnotetext{
${ }^{1}$ The negative $\Delta m_{\text {tree }}$ has been used in exotic collider phenomenology of Higgsinos [22, 23].
} 


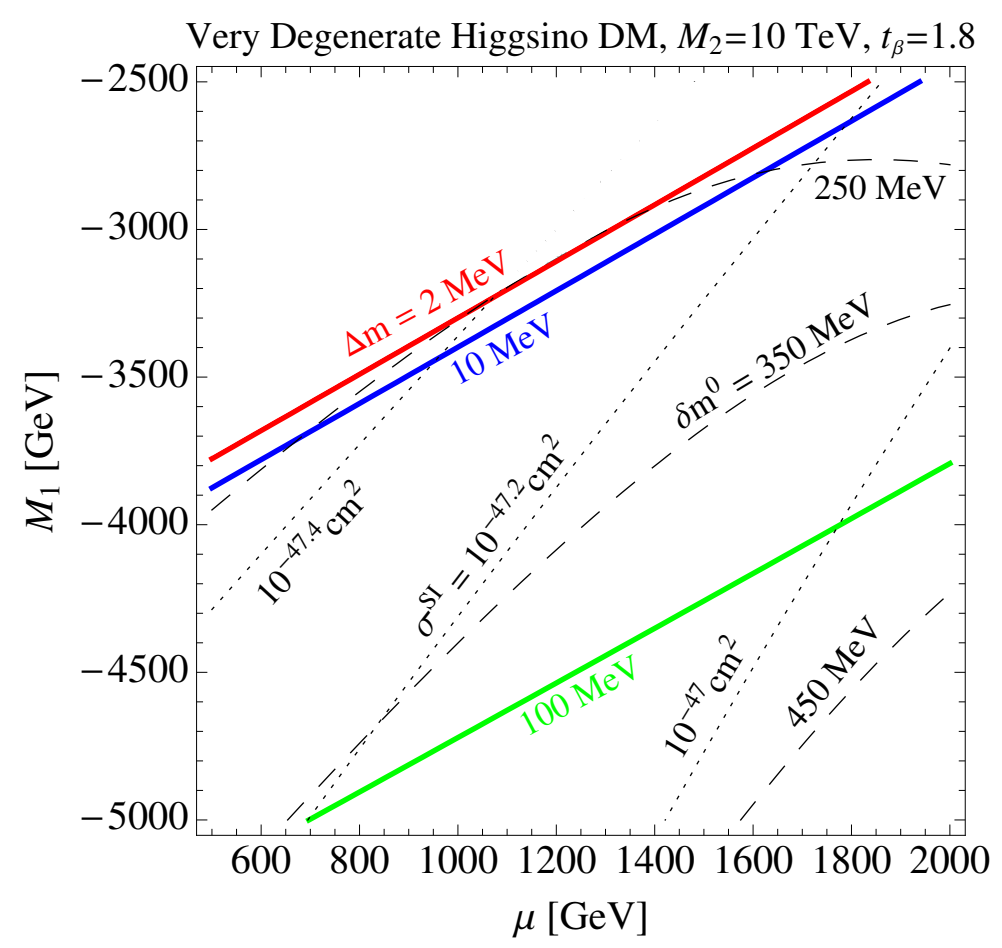

Figure 1. Very Degenerate Higgsino DM parameter space with a benchmark $M_{2}=10 \mathrm{TeV}$ and $t_{\beta}=1.8$. Contours of the mass splitting $\Delta m=m_{\chi^{+}}-\left|m_{\chi_{1}^{0}}\right|$ (solid), $\delta m^{0}=\left|m_{\chi_{2}^{0}}\right|-\left|m_{\chi_{1}^{0}}\right|$ (dashed), and spin-independent direct detection rate $\sigma^{\mathrm{SI}}$ (dotted; see section 4.1) are shown. We consider the two benchmark models along the $\Delta m=2,10 \mathrm{MeV}$ contours throughout.

\section{Indirect detection of annihilation signals}

Non-perturbative effects in DM pair annihilation can lead to Sommerfeld enhancement [13, 14] or Ramsauer-Townsend suppression [9, 11]. The pure Higgsino DM with $\mu \sim 1 \mathrm{TeV}$ and $\Delta m \approx 355 \mathrm{MeV}$ does not experience large SRT effects. Only Higgsinos as heavy as $\sim 7 \mathrm{TeV}$ can experience sizable effects, but they are too heavy to be relevant to collider experiments. On the other hand, the 1-3 TeV pure Wino DM with $\Delta m \approx 164 \mathrm{MeV}$ experiences much larger SRT effects with a resonance appearing at around $2.4 \mathrm{TeV}[8-11,13,14,17]$. Since the SRT effects on the pure Wino DM saturate at relatively high velocities $v / c \sim 10^{-2}$, Wino annihilation cross-sections at various astronomical sites with different velocity dispersions are same.

We will discuss that the very small splitting of the Higgsino DM can make the relevant Higgsino mass scale down to $\sim 1 \mathrm{TeV}$ and allow different annihilation cross-sections at various astronomical sites, postponing the saturation to lower velocities. Furthermore, there can appear not only Sommerfeld enhancements but also RT suppressions.

\subsection{SRT effects with very small mass splitting}

We focus on today's DM annihilation cross-sections into $W W, Z Z, \gamma \gamma, Z \gamma$ channels. Thus, we do not consider co-annihilation channels. Pair annihilations with SRT effects can pro- 
ceed via various intermediate two-body states with the same charge $Q=0$ and $\operatorname{spin} S=0,1$ as those of the initial LSP pair, which are exchanged by photons and on/off-shell $W, Z$ gauge bosons. We take into account all two-body states formed among Higgsino states; in addition, we add heavier gauginos if their masses are within $10 \mathrm{GeV}$ of the Higgsino in order to accommodate non-zero effects from them, but this rarely happens in our study. We follow a general formalism developed for SUSY in ref. [24-27] to calculate absorptive Wilson coefficients and non-relativistic potentials between various two-body states, and we numerically solve resulting Schrödinger equations to obtain SRT effects.

We study two benchmark models with $\Delta m=2,10 \mathrm{MeV}$ presented in figure 1. For the given $\mu \in\{600,2000\} \mathrm{GeV}$ (and other parameters as described), a unique solution for $M_{1}$ is found. As long as gaugino mixtures are small, the exact value of $M_{2}\left(\gtrsim\left|M_{1}\right|\right)$ does not matter much in annihilation signals. It is because leading contributions to annihilations and SRT effects already exist in the pure Higgsino model with vanishing gaugino mixings: for example, the direct annihilation $\chi^{0} \chi^{0} \rightarrow W W$ and the SRT effect $\chi^{0} \chi^{0} \rightarrow \chi_{1}^{+} \chi^{-}$can be mediated by the Higgsino-Higgsino- $W$ interaction without need for any gaugino mixtures. Thus, we set $M_{2}=10 \mathrm{TeV}$ ( and $t_{\beta}=1.8$ ) in this section.

In figure 2, we show contours of the annihilation cross-section into photon-line signals, $\sigma v_{\gamma \gamma+\frac{1}{2} \gamma Z} \equiv \sigma v_{\gamma \gamma}+\frac{1}{2} \sigma v_{\gamma Z}$, for the benchmark models with $\Delta m=2,10 \mathrm{MeV}$ and the usual pure Higgsino model with $\Delta m=355 \mathrm{MeV}$ for comparison. Similar features exist in photon-continuum signals from $\sigma v_{W W+Z Z} \equiv \sigma v_{W W}+\sigma v_{Z Z}$, and similar discussions apply.

Two types of enhancements are observed, most clearly from the $\Delta m=2 \mathrm{MeV}$ result. First, a series of threshold zero-energy resonances forms just below the excitation threshold of $\chi^{0} \chi^{0} \rightarrow \chi^{+} \chi^{-}$with $\frac{1}{2} \mu v^{2} \simeq \Delta m$ (blue-dashed line) [27-29], depicted as diagonal bands of enhancement. Photon exchanges between chargino pairs are responsible for the series of closely-located resonances, but not all of them are captured and shown in the figure; see ref. [27] for a demonstration of many closely-located threshold resonances. Well below the threshold, SRT effects are independent on the DM velocity as the $W$-boson exchange in $\chi^{0} \chi^{0} \rightarrow \chi^{+} \chi^{-}$becomes governed by the $W$-mass rather than the DM momentum [13, 14, 27], depicted as vertical regions of enhancement. The SRT effect saturates at finite enhancement in the $v \rightarrow 0$ limit because of the finite-ranged $W$-exchange Yukawa potential.

As $\Delta m$ increases, the excitation $\chi^{0} \chi^{0} \rightarrow \chi^{+} \chi^{-}$becomes harder and the attractive potential becomes effectively shallower [14]. A heavier DM with a smaller Bohr radius can compensate this trend and can form zero-energy bound states. Thus, the larger $\Delta m$, the heavier Higgsino Sommerfeld peaks. From $\mu \sim 1.1 \mathrm{TeV}$ for $\Delta m=2 \mathrm{MeV}$, the Sommerfeld peak moves to a heavier $\mu \sim 1.3 \mathrm{TeV}$ for $\Delta m=10 \mathrm{MeV}$ and to much heavier $\mu \sim 7 \mathrm{TeV}$ for the pure Higgsino with $\Delta m=355 \mathrm{MeV}$. Moreover, the threshold velocity becomes higher with the larger $\Delta m$, making the SRT effects saturate at higher velocities. All such behaviors are clearly shown in figure 2 .

Another remarkable is that RT dips are formed near Sommerfeld peaks $[9,11,18,19]$ both near the excitation threshold and in the small-velocity saturation regime. RT dips are located at slightly heavier Higgsino masses and/or larger velocities. As $\Delta m$ increases, dips and peaks become more separated in $\mu$ and $v$. 

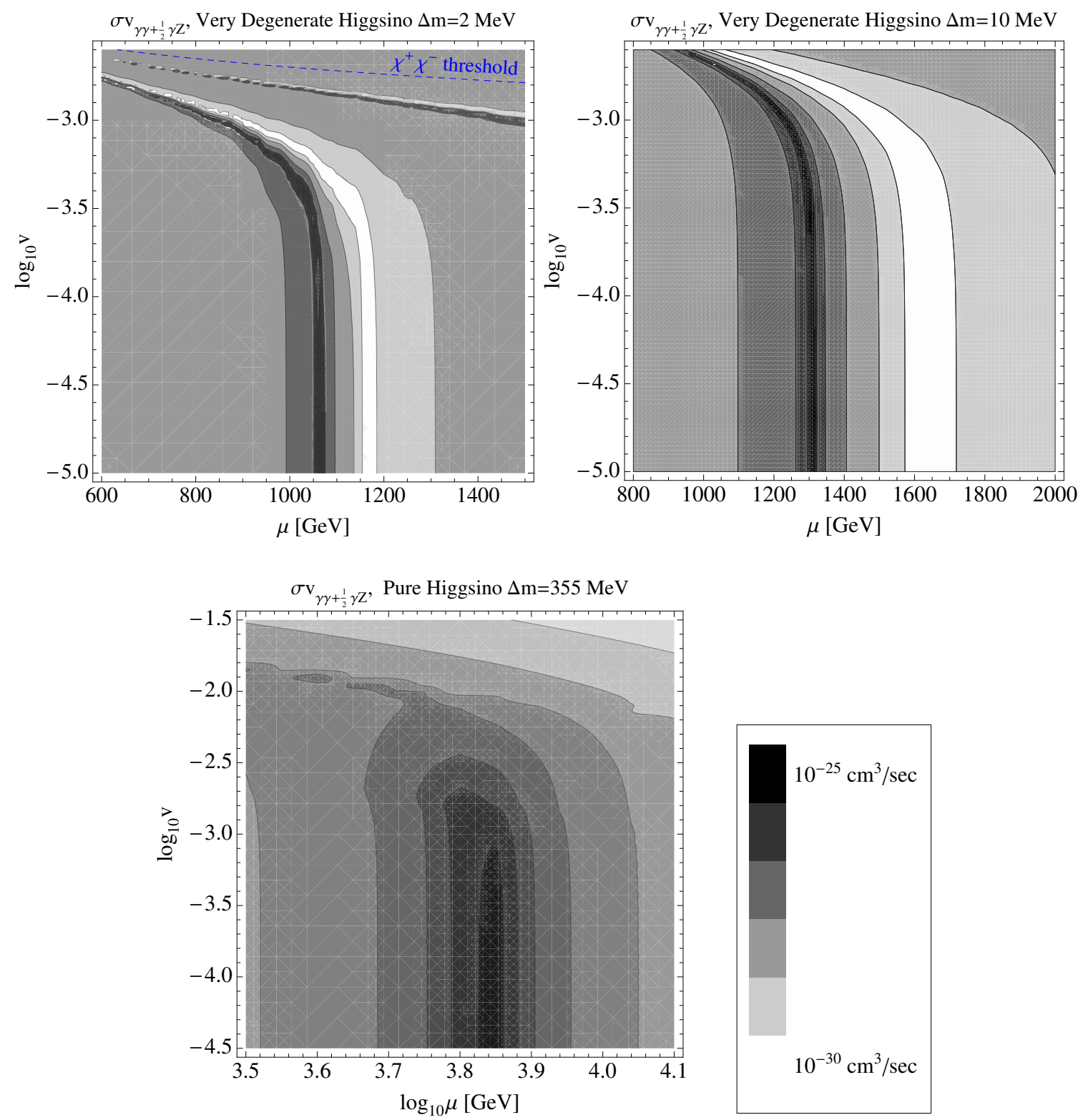

Figure 2. Contours of the annihilation cross-section into photon-line signals $\sigma v_{\gamma \gamma+\frac{1}{2} \gamma Z}$ for $\Delta m=2$ (top-left), 10 (top-right), 355 (bottom) MeV. Both enhancements from threshold resonances and RT suppressions are visible; far below the excitation threshold (blue-dashed), the SRT effects become velocity-independent. As $\Delta m$ increases, peaks and dips move to heavier masses and larger velocities, and they become more separated. Some irregularities in contours are owing to the lack of resolution in our parameter scanning. Very Degenerate Higgsino DM parameters are as in figure 1. 


\subsection{Annihilations at GC and DG}

We calculate annihilation cross-sections at GC and DG, main candidate sites for DM indirect detection. The GC is expected to support a huge DM density but also plenty of contaminations from baryons, whereas DG are very clean DM sources in spite of smaller DM density. In addition, velocity dispersions are an order of magnitude different, often further differentiating annihilation signals at DG and GC.

We convolute the annihilation cross-section calculated in the previous subsection with the Maxwell-Boltzmann velocity distributions for GC and DG [9, 30-32]

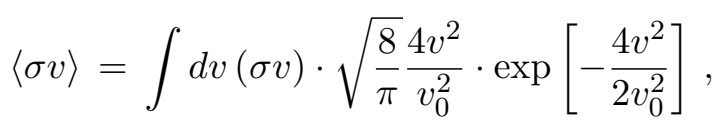

which we write in terms of the DM velocity $v=v_{\mathrm{DM}}$ (in accordance with the SRT calculation in the previous subsection) instead of the relative velocity $v_{\text {rel }}=2 v_{\mathrm{DM}}$. The velocity dispersions are chosen to be $v_{0}=210 \mathrm{~km} / \mathrm{s}$ for GC and $20 \mathrm{~km} / \mathrm{s}$ for DG. Most relevant velocity ranges are $\log _{10} v=-3.2 \sim-3.5$ for GC and $\log _{10} v=-4.1 \sim-4.6$ for DG.

Resulting velocity-convoluted annihilation cross-sections at GC and DG are shown in figure 3. Sommerfeld enhancements and RT suppressions are both clearly observed near the $1 \mathrm{TeV}$ Higgsino. Near Sommerfeld peaks and RT dips, annihilation cross-sections at GC and DG are different in general. The difference is larger for the $\Delta m=2 \mathrm{MeV}$ case because SRT effects saturate at lower velocities. Meanwhile, overall enhancements and suppressions are larger for the $\Delta m=10 \mathrm{MeV}$ case because peaks and dips are more separated in $\mu$ and $v$ so that they lead to less cancellation in the velocity convolution. We also comment that GC cross-sections are not as sharp as DG ones in the figure because we had to average over very closely-separated peaks and dips appearing just below the excitation threshold (where the GC signal is most sensitive too), not all of which is well captured in our parameter scanning.

Another remarkable feature in figure 3 is that, owing to RT dips, the DG annihilation cross-section can be smaller than that of GC. It is a counter-example to the typical result that the DG annihilation cross-section is similar or larger because the DM velocity dispersion is smaller. The existence of RT dips is (accidentally) more clear in the photon-line signal than in the photon-continuum signal; as RT dips are produced from cancellations between various contributions (not necessarily related to resonances), their appearances and strengths can depend on annihilation channels.

However, the photon-line dip depth that will be observed at detectors is subject to the internal bremsstrahlung effect. Within detector resolutions of the photon energy, the photons radiated off the $W W$ annihilation process can contribute to photon-line signals, and this extra contribution can smooth the RT-dips in figure 4. As shown in the 5plet and 7-plet DM cases studied in ref. [19], photon-line dips at some DM masses can disappear due to this extra contribution. For a better estimation of indirect detection, it is worthwhile to carry out a similar study for our doublet case; so our conclusions are subject to this uncertainty.

The peak heights shown in the figure may also be subject to uncertainties; our parameter scanning resolution very close to peak centers is limited, and perturbative cor- 

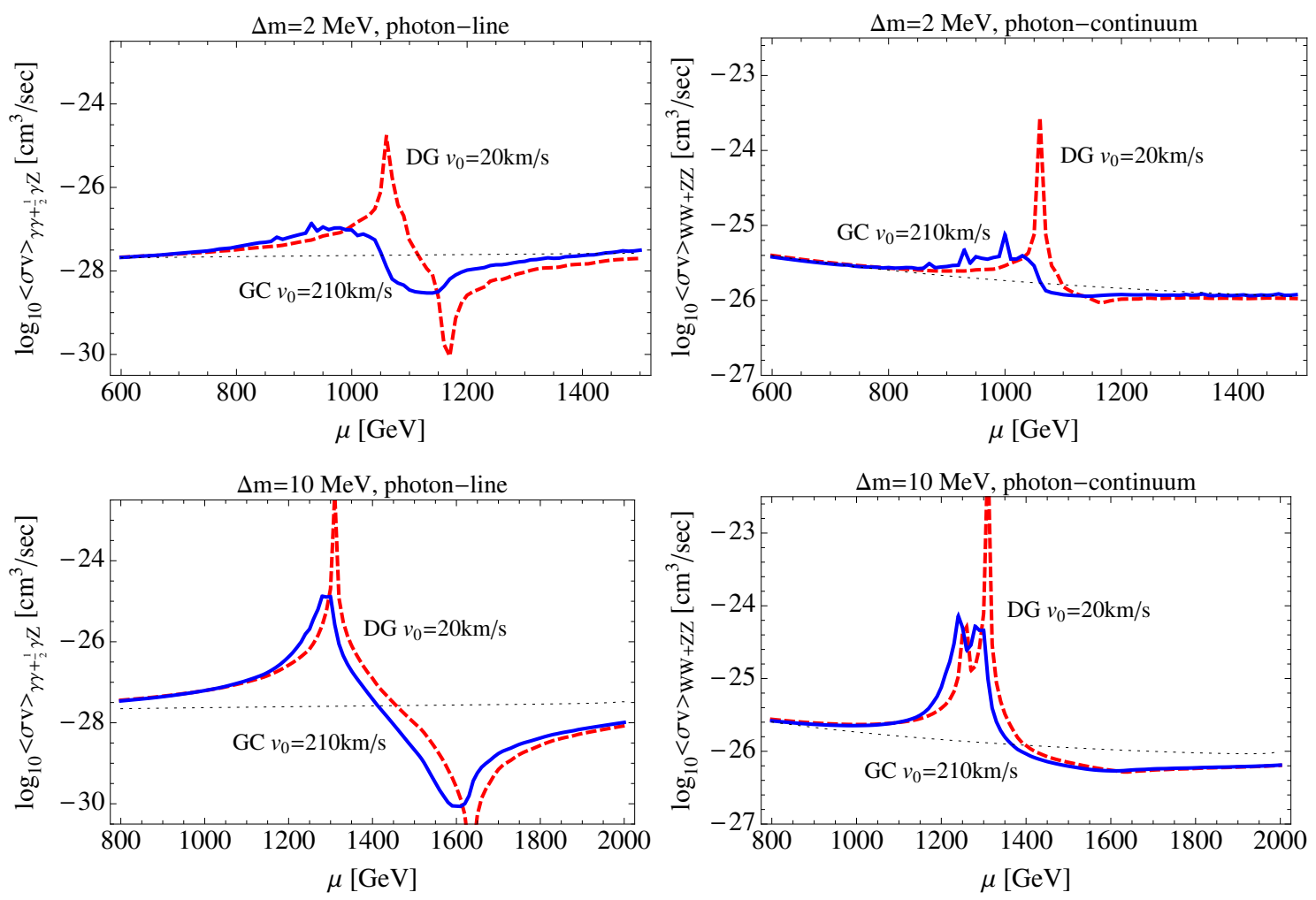

Figure 3. Annihilation cross-sections convoluted with the Maxwell-Boltzmann velocity distributions. Velocity dispersions for GC (blue-solid) and DG (red-dashed) are $v_{0}=210$ and $20 \mathrm{~km} / \mathrm{s}$. Panels are for $\Delta m=2$ (top), $10 \mathrm{MeV}$ (bottom) and the photon-line cross-section $\sigma v_{\gamma \gamma+\frac{1}{2} \gamma Z}$ (left), the photon-continuum cross-section $\sigma v_{W W+Z Z}$ (right). For comparison, perturbative results are also shown (dotted). Some irregularities are owing to the lack of resolution in our parameter scanning.

rections that may become important in this regime are not added. The perturbative corrections are most important when the unitarity is broken by unphysically enhanced cross-sections [33]. However, our annihilation cross-sections are well below the unitarity bound $\sigma v \leq 4 \pi /\left(\mu^{2} v\right) \simeq 10^{-20} \times\left(\frac{1 \mathrm{TeV}}{\mu}\right)^{2}\left(\frac{10^{-2}}{v}\right) \mathrm{cm}^{3} / \mathrm{sec} ;$ and indeed, the regularizing velocity $v_{c} \sim 10^{-6}[33]$ is much smaller than our saturation velocity. Also, our scanning resolution is good enough just away from peak centers. Thus, we do not attempt to further improve the peak height calculation.

In figure 4, we finally overlay the latest constraints and some projection limits of indirect detections. Datasets presented include: HESS 2013 [34] and Fermi-LAT 2015 [35] for photon-lines from GC, MAGIC 2013 [36] for photon-lines from DG, Fermi-LAT+MAGIC combination [37] for photon-continuum from DG, and HESS 254h [38-40] for photoncontinuum from GC. Projection studies include: CTA 5h [11] for photon-lines from GC (see refs. [41, 42] for similar results), CTA 500h [43, 44] for photon-continuum from GC, and Fermi-LAT 15 years for photon-continuum from 16 DG [45] (see ref. [46] for CTA projections). Current and future DES constraints from DG photon-continuum [47] are similar or weaker than the results shown, so we do not show them. A full DM relic density is as- 

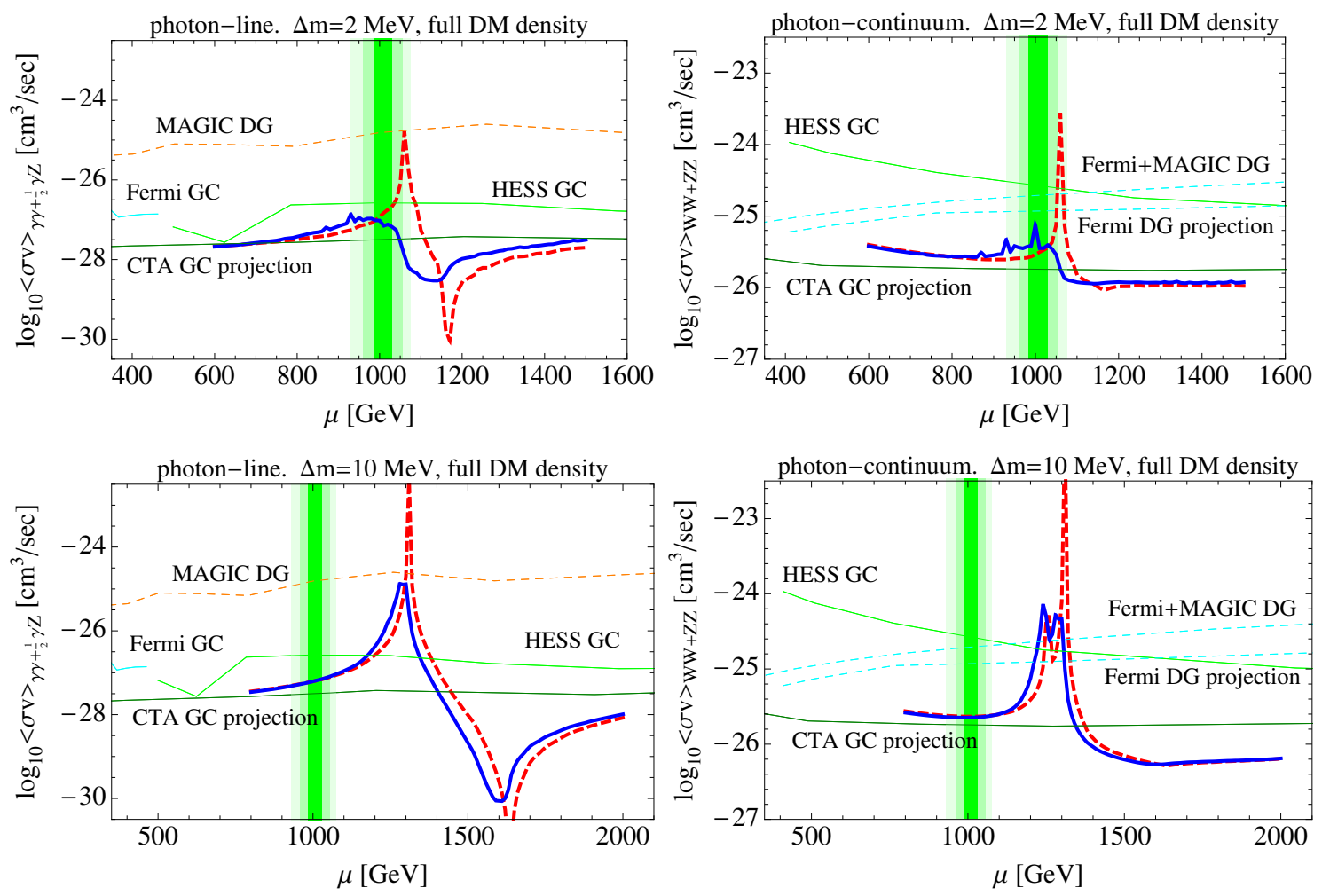

Figure 4. Latest constraints from Fermi-LAT (cyan), HESS (light green), MAGIC (orange) and projections from Fermi-LAT (cyan) and CTA (dark green); more details in text. Panels are for $\Delta m=2$ (top) and $10 \mathrm{MeV}$ (bottom), and photon-line (left) and photon-continuum (right) signals. Solid lines are for GC and dashed for DG. A full DM relic density is assumed; for reference, the usual thermal Higgsino DM mass range is shown as green bands. The internal bremsstrahlung, which can be relevant to photon-line signals, is not added.

sumed for all Higgsino masses to interpret these data as the constraints on the annihilation cross-sections.

Currently, Sommerfeld peaks in both $\Delta m=2,10 \mathrm{MeV}$ models are constrained by DG searches. Also, GC searches constrain Sommerfeld peaks of the $\Delta m=10 \mathrm{MeV}$ case, while smaller peaks of the $\Delta m=2 \mathrm{MeV}$ are not yet constrained by GC searches. In the future, a large part of the Sommerfeld enhanced parameter space can be probed by CTA GC and Fermi DG searches. On the other hand, RT dips in photon-line signals are below future sensitivities although potential positive contributions from the internal bremsstrahlung can change this somewhat. RT dips in photon-continuum signals are less significant and close to CTA GC projections.

For reference, we also show as green bands the mass range where the thermal Higgsino DM with $\Delta m=355 \mathrm{MeV}$ can explain the full DM relic density. Although SRT effects on the Very Degenerate Higgsino model can alter the relic density somewhat, the pure Higgsino result is still a useful guide as SRT effects on relic density may not be so significant; not only nearby Sommerfeld peaks and RT dips may cancel each other during a thermal history, but also some co-annihilation channels may have opposite SRT effects (as for the pure Higgsino 
DM [27]) that can also nullify impacts on the relic density. Without dedicated relic density calculations, we are content with assuming a full DM relic density which may come, e.g., from a non-thermal origin, and in any case our signals can be scaled in proportion to a true relic density.

\section{Other constraints}

\subsection{Direct detection}

The spin-independent direct detection (SIDD) signal of the nearly degenerate Higgsino DM depends on the mass splitting between the neutral states $\delta m^{0}$ and the amount of the gaugino mixture. The neutral mass gap $\delta m^{0}$ should be larger than $\mathcal{O}(0.1) \mathrm{MeV}$, otherwise its inelastic scattering mediated by the $Z$ exchange should have been already observed [9]. For the sufficiently large $\delta m^{0}$ as in our study (see figure 1), the elastic scattering rate is controlled by gaugino mixtures (via Higgsino-gaugino-Higgs coupling), that is, the signal vanishes in the pure Higgsino limit. Therefore, we consider two benchmark values of $M_{2}=10$ and $5 \mathrm{TeV}$ in this subsection, representing the cases with relatively small and large gaugino mixings and SIDD signals. For each $M_{2}$ benchmark, the value of $M_{1}$ is fixed (as a function of other parameters) to obtain the desired $\Delta m=2,10 \mathrm{MeV}$, and thus SIDD rates are determined.

The SIDD cross-section is approximately given by [48]

$$
\begin{aligned}
\sigma_{\mathrm{SI}} & \simeq 8 \times 10^{-47}\left(\frac{g_{h \chi \chi}}{0.01}\right)^{2} \mathrm{~cm}^{2}, \quad \mathcal{L} \ni g_{h \chi \chi} \overline{\chi_{1}^{0}} \chi_{1}^{0} h \\
g_{h \chi \chi} & =g\left(N_{12}-t_{W} N_{11}\right)\left(N_{14} s_{\beta}-N_{13} c_{\beta}\right) \simeq \mp g \frac{m_{W}}{2 M_{2}}\left(1 \mp s_{2 \beta}\right)\left(\epsilon_{K} \mp \frac{\mu}{M_{1}}\left(1+t_{W}^{2}\right)\right),
\end{aligned}
$$

where the sign $\mp$ implies the sign $\left(-\epsilon_{K}\right)$ and we assume the Higgs alignment limit. We obtain $\sigma_{\mathrm{SI}}=(3 \sim 5) \times 10^{-48},(4 \sim 9) \times 10^{-47} \mathrm{~cm}^{2}$ for the $M_{2}=10,5 \mathrm{TeV}$ with the range spanned by $\mu=600 \sim 1500 \mathrm{GeV}$ (see figure 1 for $M_{2}=10 \mathrm{TeV}$ result). The dependence on the $\Delta m$ (indirectly via Bino mixtures) is not significant for $\Delta m \lesssim 10 \mathrm{MeV}$. The former range of $\sigma_{\mathrm{SI}}$ with $M_{2}=10 \mathrm{TeV}$ is close to the coherent neutrino scattering background floor so that searches will be difficult in the near future, while the latter range with $M_{2}=5 \mathrm{TeV}$ is expected to be probed at future experiments such as DarkSide-G2 [49, 50] and LZ [49, 51]. Although indirect detection signals are sizable for both $M_{2}$ benchmark values, the absence or existence of detectable SIDD signals still depends on the Wino mixture (hence, the Wino mass), and either is not a necessary consequence of the Very Degenerate Higgsino DM.

Meanwhile, more interesting direct detection signals of our model can be produced by the formation of a DM-nucleus bound state through the inelastic scattering of $\chi^{0} N_{Z} \rightarrow$ $\chi^{-} N_{Z+1}[52-54]$. The latest analysis adopting a semi-classical calculation in the Fermi gas model of nuclei [54] showed that neutrinoless double-beta decay experiments like EXO200 and Kamland-Zen are able to provide a unique and strong sensitivity to the model parameter space with $\Delta m$ smaller than the chargino-nucleus binding energy $\sim 20 \mathrm{MeV}$. Further progresses in understanding nuclear model dependences of the nuclear transition element and/or improving experimental sensitivies will be crutial to test our model. 


\subsection{Collider searches}

With the very small mass splitting, the charged Higgsino can be long-lived at LHC experiments. If it decays outside or the outer part of LHC detectors, stable chargino searches can apply, that is, characteristic ionization patterns of traversing massive charged particles can be identified. If it decays in the middle of detectors, disappearing charged track searches can apply as soft charged decay products are not efficiently reconstructed.

For $\Delta m$ much smaller than the pion mass, the dominant chargino decay mode is $\chi^{+} \rightarrow e^{+} \nu_{e} \chi^{0}[12,55-57]:$

$$
\Gamma\left(\chi^{+} \rightarrow e^{+} \nu_{e} \chi^{0}\right)=\frac{G_{F}^{2}}{30 \pi^{3}}(\Delta m)^{5} \sqrt{1-\left(\frac{m_{e}}{\Delta m}\right)^{2}} P\left(m_{e} / \Delta m\right)
$$

with the function $P(x)$ given in ref. [12]. For $\Delta m \sim \mathcal{O}(1-10) \mathrm{MeV}$, the decay length is very long, $c \tau \sim 10^{7}-10^{12} \mathrm{~m}$ (equivalently $\tau \sim 10^{-1}-10^{4} \mathrm{sec}$ ), so that almost all charginos traverse LHC detectors and thus only stable chargino searches apply.

By reinterpreting the CMS $8 \mathrm{TeV}$ constraints on the stable charged pure Wino [58], we obtain the constraint $\mu \gtrsim 400-600 \mathrm{GeV}$ for $\Delta m$ much smaller than the pion mass. The uncertainty range quoted is partly owing to the lack of our knowledge of $r_{\min }$, the minimum decay length of the chargino for the CMS stable chargino search to be applied; it is needed because CMS considered the range of charged Wino decay length $c \tau=\mathcal{O}(0.1-10) \mathrm{m}$ where only a fraction of charged Winos traverse detectors and become stable charginos. From the CMS acceptance curve in ref. [58], we choose to vary $r_{\min }=c \tau_{\min } \simeq 1.5-6 \mathrm{~m}$ $\left(\tau_{\min }=5-20 \mathrm{~ns}\right)$ to obtain the constraint and uncertainty.

We conclude that the $\sim 1 \mathrm{TeV}$ Very Degenerate Higgsino DM is currently allowed, but future LHC searches of stable charginos will better constrain the model.

\subsection{Cosmological constraints}

The long-lived charged Higgsino can be cosmologically dangerous. The above quoted lifetime in our model $\tau \sim 10^{-1}-10^{4}$ sec could endanger the standard bing-bang nucleosynthesis $(\mathrm{BBN})$ prediction. Although the chargino decay releases only soft leptons not directly affecting BBN, its metastable existence can form a bound state with a helium and can catalyze the ${ }^{6} \mathrm{Li}$ production. The lifetime limit $\tau \lesssim 5000$ sec of such a metastable charged particle [59] constrains the Higgsino mass splitting to be $\Delta m \gtrsim 1.2 \mathrm{MeV}{ }^{2}$ The $(\Delta m)^{5}$ dependence of the decay width in eq. (4.3) makes the BBN constraints quickly irrelevant to larger $\Delta m$ cases that we focus on.

As the enhancement is saturated at modestly small velocity, early-universe constraints from the era with the very small DM velocity such as recombination and DM protohalo formation are not strong. For example, $\sigma v_{W W} \lesssim 10^{-24} \mathrm{~cm}^{3} / \mathrm{sec}$ is generally safe from such considerations (see, e.g., refs. [60-62]), so that the model is not constrained possibly except for very small parameter spaces close to Sommerfeld peaks.

\footnotetext{
${ }^{2}$ The limit on stau-neutralino mass splitting, $70 \mathrm{MeV}$, reported in ref. [59] is much stronger because the stau has four-body decays and is thus longer-lived.
} 


\section{Summary and discussions}

We have studied the Very Degenerate Higgsino DM model with $\mathcal{O}(1) \mathrm{MeV}$ mass splitting, which is realized by small gaugino mixings and leads to dramatic non-perturbative effects. Owing to the very small mass splitting, SRT peaks and dips are present at around the $1 \mathrm{TeV}$ Higgsino mass, and the velocity saturation of SRT effects is postponed to lower velocities $v / c \sim 10^{-3}$. As a result, indirect detection signals of $\sim 1 \mathrm{TeV}$ Higgsino DM can be significantly Sommerfeld-enhanced (to be constrained already or observable in the near future) or even RT-suppressed. Annihilation cross-sections at GC and DG are different in general: either of them can be larger than the other depending on the locations of Sommerfeld peaks and RT dips. But our conclusions are subject to unaccounted internal bremsstrahlung effects which can smooth RT dips. Further studies are required to check that our results are robust. Meanwhile, other observable signature is also induced in stable chargino collider searches, which can probe the $1 \mathrm{TeV}$ scale in the future. However, the rates of direct detection signals depend on the $M_{2}$ value (the smaller $M_{2}$, the larger signal) so that $M_{2} \sim 5(10) \mathrm{TeV}$ can(not) produce detectable signals. The potentially unusual aspects of indirect detection signals discussed in this paper are well featured by the two benchmark models of $\Delta m=2$ and $10 \mathrm{MeV}$ and shall be well taken into account in future searches and interpretations in terms of Higgsino DM models.

The Very Degenerate Higgsino DM also provides an example where "slight" gaugino mixings can have unexpectedly big impacts on the observation prospects of the Higgsino DM. The mixing is slight in the sense that the direct detection, whose leading contribution is induced by gaugino mixings, can still be small (for heavy enough Winos). But the phenomenology is unexpectedly interesting because such small mixings are usually thought not to affect the indirect detection signal, as the signal is already sizable in the zeromixing limit. In all, nearly pure Higgsino DM can have vastly different phenomena and discovery prospects from the pure Higgsino DM, and we hope that more complete studies can be followed.

\section{Acknowledgments}

We thank Kyu Jung Bae and Ranjan Laha for discussions on cosmological constraints, Kfir Blum on regulating Sommerfeld peaks, and Haipeng An on chargino-nucleus bound states. The work of SJ is supported by the US Department of Energy under contract DE-AC02-76SF00515 and in part by the National Science Foundation under Grant No. NSF PHY11-25915. JCP is supported by the Basic Science Research Program through the National Research Foundation of Korea (NRF-2013R1A1A2061561, 2016R1C1B2015225). SJ thanks KITP for their hospitality during the completion of the work.

Open Access. This article is distributed under the terms of the Creative Commons Attribution License (CC-BY 4.0), which permits any use, distribution and reproduction in any medium, provided the original author(s) and source are credited. 


\section{References}

[1] N. Arkani-Hamed, A. Delgado and G.F. Giudice, The Well-tempered neutralino, Nucl. Phys. B 741 (2006) 108 [hep-ph/0601041] [INSPIRE].

[2] M. Low and L.-T. Wang, Neutralino dark matter at $14 \mathrm{TeV}$ and $100 \mathrm{TeV}$, JHEP 08 (2014) 161 [arXiv: 1404.0682] [INSPIRE].

[3] B.S. Acharya, K. Bozek, C. Pongkitivanichkul and K. Sakurai, Prospects for observing charginos and neutralinos at a $100 \mathrm{TeV}$ proton-proton collider, JHEP 02 (2015) 181 [arXiv: 1410.1532] [INSPIRE].

[4] S. Gori, S. Jung, L.-T. Wang and J.D. Wells, Prospects for Electroweakino Discovery at a 100 TeV Hadron Collider, JHEP 12 (2014) 108 [arXiv:1410.6287] [InSPIRE].

[5] D. Barducci, A. Belyaev, A.K.M. Bharucha, W. Porod and V. Sanz, Uncovering Natural Supersymmetry via the interplay between the LHC and Direct Dark Matter Detection, JHEP 07 (2015) 066 [arXiv: 1504.02472] [INSPIRE].

[6] M. Badziak, A. Delgado, M. Olechowski, S. Pokorski and K. Sakurai, Detecting underabundant neutralinos, JHEP 11 (2015) 053 [arXiv: 1506.07177] [INSPIRE].

[7] J. Bramante, N. Desai, P. Fox, A. Martin, B. Ostdiek and T. Plehn, Towards the Final Word on Neutralino Dark Matter, Phys. Rev. D 93 (2016) 063525 [arXiv:1510.03460] [InSPIRE].

[8] M. Cirelli, A. Strumia and M. Tamburini, Cosmology and Astrophysics of Minimal Dark Matter, Nucl. Phys. B 787 (2007) 152 [arXiv:0706.4071] [INSPIRE].

[9] E.J. Chun, J.-C. Park and S. Scopel, Non-perturbative Effect and PAMELA Limit on Electro-Weak Dark Matter, JCAP 12 (2012) 022 [arXiv:1210.6104] [INSPIRE].

[10] J. Fan and M. Reece, In Wino Veritas? Indirect Searches Shed Light on Neutralino Dark Matter, JHEP 10 (2013) 124 [arXiv:1307.4400] [INSPIRE].

[11] E.J. Chun and J.-C. Park, Electro-Weak Dark Matter: non-perturbative effect confronting indirect detections, Phys. Lett. B 750 (2015) 372 [arXiv:1506.07522] [INSPIRE].

[12] S.D. Thomas and J.D. Wells, Phenomenology of Massive Vectorlike Doublet Leptons, Phys. Rev. Lett. 81 (1998) 34 [hep-ph/9804359] [INSPIRE].

[13] J. Hisano, S. Matsumoto and M.M. Nojiri, Explosive dark matter annihilation, Phys. Rev. Lett. 92 (2004) 031303 [hep-ph/0307216] [INSPIRE].

[14] J. Hisano, S. Matsumoto, M.M. Nojiri and O. Saito, Non-perturbative effect on dark matter annihilation and gamma ray signature from galactic center, Phys. Rev. D 71 (2005) 063528 [hep-ph/0412403] [INSPIRE].

[15] M. Cirelli, F. Sala and M. Taoso, Wino-like Minimal Dark Matter and future colliders, JHEP 10 (2014) 033 [Erratum ibid. 01 (2015) 041] [arXiv: 1407.7058] [INSPIRE].

[16] B. Bhattacherjee, B. Feldstein, M. Ibe, S. Matsumoto and T.T. Yanagida, Pure gravity mediation of supersymmetry breaking at the Large Hadron Collider, Phys. Rev. D 87 (2013) 015028 [arXiv: 1207.5453 ] [INSPIRE].

[17] T. Cohen, M. Lisanti, A. Pierce and T.R. Slatyer, Wino Dark Matter Under Siege, JCAP 10 (2013) 061 [arXiv:1307.4082] [INSPIRE].

[18] M. Cirelli, T. Hambye, P. Panci, F. Sala and M. Taoso, Gamma ray tests of Minimal Dark Matter, JCAP 10 (2015) 026 [arXiv:1507.05519] [INSPIRE]. 
[19] C. Garcia-Cely, A. Ibarra, A.S. Lamperstorfer and M.H.G. Tytgat, Gamma-rays from Heavy Minimal Dark Matter, JCAP 10 (2015) 058 [arXiv:1507.05536] [INSPIRE].

[20] M. Drees, M.M. Nojiri, D.P. Roy and Y. Yamada, Light Higgsino dark matter, Phys. Rev. D 56 (1997) 276 [Erratum ibid. D 64 (2001) 039901] [hep-ph/9701219] [INSPIRE].

[21] N. Nagata and S. Shirai, Higgsino Dark Matter in High-Scale Supersymmetry, JHEP 01 (2015) 029 [arXiv: 1410.4549] [INSPIRE].

[22] G.D. Kribs, A. Martin and T.S. Roy, Supersymmetry with a Chargino NLSP and Gravitino LSP, JHEP 01 (2009) 023 [arXiv:0807.4936] [INSPIRE].

[23] S. Jung and H.-S. Lee, Untracked Signals of Supersymmetry at the LHC, arXiv:1503.00414 [INSPIRE].

[24] M. Beneke, C. Hellmann and P. Ruiz-Femenia, Non-relativistic pair annihilation of nearly mass degenerate neutralinos and charginos I. General framework and S-wave annihilation, JHEP 03 (2013) 148 [Erratum ibid. 10 (2013) 224] [arXiv:1210.7928] [INSPIRE].

[25] C. Hellmann and P. Ruiz-Femenía, Non-relativistic pair annihilation of nearly mass degenerate neutralinos and charginos II. P-wave and next-to-next-to-leading order S-wave coefficients, JHEP 08 (2013) 084 [arXiv: 1303.0200] [INSPIRE].

[26] M. Beneke, C. Hellmann and P. Ruiz-Femenia, Non-relativistic pair annihilation of nearly mass degenerate neutralinos and charginos III. Computation of the Sommerfeld enhancements, JHEP 05 (2015) 115 [arXiv: 1411.6924] [INSPIRE].

[27] M. Beneke, C. Hellmann and P. Ruiz-Femenia, Heavy neutralino relic abundance with Sommerfeld enhancements - a study of pMSSM scenarios, JHEP 03 (2015) 162 [arXiv: 1411.6930] [INSPIRE].

[28] T.R. Slatyer, The Sommerfeld enhancement for dark matter with an excited state, JCAP 02 (2010) 028 [arXiv:0910.5713] [inSPIRE].

[29] J.D. March-Russell and S.M. West, WIMPonium and Boost Factors for Indirect Dark Matter Detection, Phys. Lett. B 676 (2009) 133 [arXiv:0812.0559] [INSPIRE].

[30] J.L. Feng, M. Kaplinghat and H.-B. Yu, Sommerfeld Enhancements for Thermal Relic Dark Matter, Phys. Rev. D 82 (2010) 083525 [arXiv:1005.4678] [INSPIRE].

[31] R. Essig, N. Sehgal, L.E. Strigari, M. Geha and J.D. Simon, Indirect Dark Matter Detection Limits from the Ultra-Faint Milky Way Satellite Segue 1, Phys. Rev. D 82 (2010) 123503 [arXiv: 1007 .4199] [INSPIRE].

[32] M. Cannoni, Relativistic $\left\langle\sigma v_{\text {rel }}>\right.$ in the calculation of relics abundances: a closer look, Phys. Rev. D 89 (2014) 103533 [arXiv:1311.4494] [INSPIRE].

[33] K. Blum, R. Sato and T.R. Slatyer, Self-consistent Calculation of the Sommerfeld Enhancement, JCAP 06 (2016) 021 [arXiv: 1603.01383] [INSPIRE].

[34] H.E.S.S. collaboration, A. Abramowski et al., Search for Photon-Linelike Signatures from Dark Matter Annihilations with H.E.S.S., Phys. Rev. Lett. 110 (2013) 041301 [arXiv: 1301.1173] [INSPIRE].

[35] FERMI-LAT collaboration, M. Ackermann et al., Updated search for spectral lines from Galactic dark matter interactions with pass 8 data from the Fermi Large Area Telescope, Phys. Rev. D 91 (2015) 122002 [arXiv:1506.00013] [INSPIRE]. 
[36] J. Aleksić et al., Optimized dark matter searches in deep observations of Segue 1 with MAGIC, JCAP 02 (2014) 008 [arXiv:1312.1535] [INSPIRE].

[37] Fermi-LAT, MAGIC collaboration, M.L. Ahnen et al., Limits to dark matter annihilation cross-section from a combined analysis of MAGIC and Fermi-LAT observations of dwarf satellite galaxies, JCAP 02 (2016) 039 [arXiv:1601.06590] [INSPIRE].

[38] H.E.S.S. collaboration, V. Lefranc and E. Moulin, Dark matter search in the inner Galactic halo with H.E.S.S. I and H.E.S.S. II, PoS (ICRC2015) 1208 [arXiv: 1509.04123] [INSPIRE].

[39] K.N. Abazajian and J.P. Harding, Constraints on WIMP and Sommerfeld-Enhanced Dark Matter Annihilation from HESS Observations of the Galactic Center, JCAP 01 (2012) 041 [arXiv: 1110.6151] [INSPIRE].

[40] H.E.S.S. collaboration, A. Abramowski et al., Search for a Dark Matter annihilation signal from the Galactic Center halo with H.E.S.S, Phys. Rev. Lett. 106 (2011) 161301 [arXiv:1103.3266] [INSPIRE].

[41] L. Bergstrom, G. Bertone, J. Conrad, C. Farnier and C. Weniger, Investigating Gamma-Ray Lines from Dark Matter with Future Observatories, JCAP 11 (2012) 025 [arXiv:1207.6773] [INSPIRE].

[42] A. Ibarra, A.S. Lamperstorfer, S. López-Gehler, M. Pato and G. Bertone, On the sensitivity of CTA to gamma-ray boxes from multi-TeV dark matter, JCAP 09 (2015) 048 [arXiv: 1503.06797] [INSPIRE].

[43] CTA collaboration, J. Carr et al., Prospects for Indirect Dark Matter Searches with the Cherenkov Telescope Array (CTA), PoS(ICRC2015)1203 [arXiv: 1508.06128] [INSPIRE].

[44] V. Lefranc, E. Moulin, P. Panci and J. Silk, Prospects for Annihilating Dark Matter in the inner Galactic halo by the Cherenkov Telescope Array, Phys. Rev. D 91 (2015) 122003 [arXiv: 1502.05064] [INSPIRE].

[45] Fermi-LAT collaboration, E. Charles et al., Sensitivity Projections for Dark Matter Searches with the Fermi Large Area Telescope, Phys. Rept. 636 (2016) 1 [arXiv:1605.02016] [INSPIRE].

[46] V. Lefranc, G.A. Mamon and P. Panci, Prospects for annihilating Dark Matter towards Milky Way's dwarf galaxies by the Cherenkov Telescope Array, JCAP 09 (2016) 021 [arXiv: 1605.02793] [INSPIRE].

[47] DES, Fermi-LAT collaborations, A. Drlica-Wagner et al., Search for Gamma-Ray Emission from DES Dwarf Spheroidal Galaxy Candidates with Fermi-LAT Data, Astrophys. J. 809 (2015) L4 [arXiv: 1503.02632] [INSPIRE].

[48] C. Cheung, L.J. Hall, D. Pinner and J.T. Ruderman, Prospects and Blind Spots for Neutralino Dark Matter, JHEP 05 (2013) 100 [arXiv:1211.4873] [INSPIRE].

[49] P. Cushman et al., Working Group Report: WIMP Dark Matter Direct Detection, arXiv: 1310.8327 [INSPIRE].

[50] C.E. Aalseth et al., The DarkSide Multiton Detector for the Direct Dark Matter Search, Adv. High Energy Phys. 2015 (2015) 541362.

[51] LZ collaboration, D.S. Akerib et al., LUX-ZEPLIN (LZ) Conceptual Design Report, arXiv: 1509.02910 [INSPIRE]. 
[52] M. Pospelov and A. Ritz, Resonant scattering and recombination of pseudo-degenerate WIMPs, Phys. Rev. D 78 (2008) 055003 [arXiv:0803.2251] [InSPIRE].

[53] Y. Bai and P.J. Fox, Resonant Dark Matter, JHEP 11 (2009) 052 [arXiv:0909.2900] [INSPIRE].

[54] H. An, M. Pospelov and J. Pradler, Direct constraints on charged excitations of dark matter, Phys. Rev. Lett. 109 (2012) 251302 [arXiv:1209.6358] [INSPIRE].

[55] C.H. Chen, M. Drees and J.F. Gunion, A Nonstandard string/SUSY scenario and its phenomenological implications, Phys. Rev. D 55 (1997) 330 [Erratum ibid. D 60 (1999) 039901] [hep-ph/9607421] [INSPIRE].

[56] S.P. Martin, Collider signals from slow decays in supersymmetric models with an intermediate scale solution to the mu problem, Phys. Rev. D 62 (2000) 095008 [hep-ph/0005116] [INSPIRE].

[57] S. Jung, Resolving the existence of Higgsinos in the LHC inverse problem, JHEP 06 (2014) 111 [arXiv: 1404.2691] [INSPIRE].

[58] CMS collaboration, Constraints on the pMSSM, AMSB model and on other models from the search for long-lived charged particles in proton-proton collisions at $\sqrt{s}=8 \mathrm{TeV}$, Eur. Phys. J. C 75 (2015) 325 [arXiv: 1502.02522] [INSPIRE].

[59] M. Pospelov, Particle physics catalysis of thermal Big Bang Nucleosynthesis, Phys. Rev. Lett. 98 (2007) 231301 [hep-ph/0605215] [INSPIRE].

[60] M. Kamionkowski and S. Profumo, Early Annihilation and Diffuse Backgrounds in Models of Weakly Interacting Massive Particles in Which the Cross Section for Pair Annihilation Is Enhanced by 1/v, Phys. Rev. Lett. 101 (2008) 261301 [arXiv:0810.3233] [INSPIRE].

[61] S. Galli, F. Iocco, G. Bertone and A. Melchiorri, CMB constraints on Dark Matter models with large annihilation cross-section, Phys. Rev. D 80 (2009) 023505 [arXiv:0905.0003] [INSPIRE].

[62] T.R. Slatyer, N. Padmanabhan and D.P. Finkbeiner, CMB Constraints on WIMP Annihilation: Energy Absorption During the Recombination Epoch, Phys. Rev. D 80 (2009) 043526 [arXiv: 0906.1197] [INSPIRE]. 